\title{
Phase I/II Study of Extreme Hypofractionated Stereotactic Body Radiation Therapy Boost to Prostate for Locally Advanced, Node-Positive and Oligometastatic Cancer
}

Kushal Narang ${ }^{1}$, Mohit Kadian ${ }^{1}$, K Venkatesan ${ }^{1}$, Saumyaranjan Mishra ${ }^{1}$, Shyam Bisht ${ }^{1}$, Deepak Gupta ${ }^{1}$, Susovan Banerjee ${ }^{1}$, Tejinder Kataria ${ }^{1}$

1. Division of Radiation Oncology, Medanta Cancer Institute, Medanta The Medicity, Gurugram, IND

Corresponding author: Kushal Narang, narangkushal@gmail.com

\begin{abstract}
Introduction: Stereotactic body radiation therapy (SBRT) is increasingly being utilized to deliver escalated radiation doses for improving outcomes in various malignancies. We analyzed our cohort of locally advanced, node-positive, and bone oligometastatic prostate cancer patients, that were treated with a combination of pelvic RT using conventional fractionation (CF) and SBRT boost to prostate using extreme hypofractionation (EH), along with hormone therapy (HT).
\end{abstract}

Materials and Methods: Outcomes of 44 prospectively treated patients were analyzed. Volumetric modulated arc therapy (VMAT) was utilized to deliver a dose of 45 Gy to pelvic nodal region, 50 Gy to prostate, and 5456 Gy to gross nodes in 25 fractions. EH boost $18 \mathrm{~Gy}$ in three fractions was delivered to the prostate using CyberKnife (Accuray, Sunnyvale, CA, USA) SBRT. Bone oligometastasis, if any, were treated to a dose of 16 Gy in two fractions, delivered on weekends. Serum prostate-specific antigen (PSA), multi-parametric magnetic resonance imaging (MRI) of pelvis, and prostate-specific membrane antigen-positron emission tomography (PSMA-PET) were used for response assessment during follow-up. HT was given as per standard guidelines.

Results: There were 33 (75\%) locally advanced, nine (20.5\%) node-positive, and two (4.5\%) oligometastatic cases. At a median follow-up of 63.5 months, the five-year progression-free survival (PFS) was $88.2 \%$, biochemical PFS (bPFS) was $91.4 \%$ and overall survival (OS) was $96.9 \%$. Grade III or greater acute genitourinary and gastrointestinal toxicity was $2.3 \%$ each, and late toxicity was $4.5 \%$ and $0 \%$, respectively.

Conclusion: Excellent five-year outcomes can be attained even for locally advanced, node-positive and bone oligometastatic prostate cancer, by means of dose-escalation using EH-SBRT boost to the prostate.

Review began 10/01/2020 Review ended 11/15/2020 Published 11/28/2020

\section{() Copyright 2020}

Narang et al. This is an open access article distributed under the terms of the Creative Commons Attribution License CC-BY 4.0., which permits unrestricted use, distribution, and reproduction in any medium, provided the original author and source are credited.
Categories: Medical Physics, Radiation Oncology, Oncology

Keywords: advanced, extreme hypofractionation, node-positive, oligometastatic, prostate, boost, sbrt

\section{Introduction}

Prostate cancer $(\mathrm{PC})$ has an alpha/beta $(\alpha / \beta)$ value of around 1.5 , which is lower than that of nearby critical structures like the bladder and rectum. This means that irradiating with doses per fraction greater than conventional fractionation (CF) would deliver a greater biologically equivalent dose (BED) to the prostate, without significantly increasing late normal tissue toxicity. The outcomes of studies employing doseescalated CF (78-81 Gy) [1] are comparable with those of moderate hypofractionation (MH; 2.4-4 Gy per fraction) [2-3]. In recent years, multiple phase two and phase three studies employing extreme hypofractionation (EH; $\geqslant 5$ Gy per fraction) have also reported excellent control rates with acceptable bladder and rectal toxicity. Although most of these studies have evaluated low-intermediate risk, localized PC [4], data for EH for high-risk, localized and advanced PC is rapidly accumulating [5-12]. However, use of this radical approach for oligometastatic cases is rarely reported. Since September 2012, we have been treating locally advanced, node-positive and bone oligometastatic PC cases with a combination of CF for prostate and pelvic nodal volume, with an EH boost to prostate and weekly hypofractionated radiation therapy (RT) for the metastatic site. We hereby report the five-year outcomes for this cohort.

\section{Materials And Methods}

We reviewed our prospectively maintained dataset of consecutively treated PC cases. From this dataset, patients with locally advanced and bone oligometastatic PC, treated with a combination of CF using volumetric modulated arc therapy (VMAT) and EH boost to prostate using CyberKnife ${ }^{\circledR}$ (CK; Accuray, Sunnyvale, CA, USA) stereotactic body radiation therapy (SBRT), were identified. Patients with less than one year of available follow-up were excluded. Thus, 44 patients, treated between August 2012 and June 2019, constituted the study dataset. 


\section{Treatment methodology}

All patients underwent complete baseline staging work-up, including clinical examination, serum prostatespecific antigen (PSA), multi-parametric magnetic resonance imaging (MRI) of the pelvis including MR spectroscopy, diffusion-weighted imaging (DWI) with absolute diffusion coefficient (ADC) mapping, 12-core biopsy of the prostate including Gleason's Score (GS) and a bone scan. Prostate-specific membrane antigen positron emission tomography (PSMA-PET) scan was available after September 2015. Staging and risk stratification was done as per National Comprehensive Cancer Network (NCCN) guidelines [13]. Bone oligometastasis was defined as presence of up to five metastatic sites. Treatment was initiated with neoadjuvant HT using bicalutamide and a luteinising hormone releasing hormone agonist (combined androgen block). After eight to 12 weeks, digital rectal examination, serum PSA and MRI pelvis were done to assess the prostate volume and prostate-rectum interface. Uroflowmetry and assessment of International Prostate Symptom Score (IPSS) were done for all patients. SBRT with EH was considered for patients with prostate volume $<50 \mathrm{cc}$ and a distinct prostate-rectum interface. Contraindications for use of EH were prior history of trans-urethral resection of prostate (TURP), and significant urinary symptoms (IPSS $\geqslant 15$ ).

\section{RT planning and delivery}

Preparation, Mould Procedure and Planning Scans

Treatment by CK-SBRT necessitated prior placement of three to four gold seed fiducial markers in the prostate for intrafraction motion tracking. After ensuring normal bleeding and clotting parameters, and under prophylactic antibiotic cover, fiducial markers of 3-5mm length and $1 \mathrm{~mm}$ diameter were placed at the apex, base and lateral edges of the prostate using trans-rectal ultrasound guidance, and their positional geometry verified by X-ray imaging on CK. The patients underwent planning scans after five days to allow for fiducial stabilization and settlement of post-procedure oedema. Patients consumed two tablets of activated charcoal every night, starting two days prior to the date of RT planning, as part of bowel preparation. On the day of planning, patients were immobilized in supine position with their arms overhead in a vacuum cushion molded to match their body contours. They were kept nil by mouth for four hours prior, and rectal emptying ensured by administering an enema one hour prior to the scan. The volume of interest was selected from first lumbar vertebra to mid-thigh region, keeping a minimum $10-15 \mathrm{~cm}$ margin above and below the target region. A non-contrast scan with $1 \mathrm{~mm}$ slice thickness with empty bladder was performed for CK-SBRT. Subsequently, three external fiducial markers were placed over the pelvis using alignment lasers and a $3 \mathrm{~mm}$ contrast-enhanced scan with mid-filled bladder was performed for the fractionated RT phase.

\section{Target Delineation}

Firstly, accessory imaging including MRI and PSMA-PET were fused with the planning CT scan image-sets. The prostate, any associated extracapsular or seminal vesicle (SV) involvement, gross nodes and oligometastatic bone lesions were marked as respective gross target volumes (GTV). The uninvolved part of seminal vesicles was not included in GTV. For VMAT utilizing daily inter-fraction imaging, a planning target volume (PTV) of $10 \mathrm{~mm}$ all around, except for $5 \mathrm{~mm}$ in posterior direction was added to the prostate GTV. These margins helped encompass the major part of seminal vesicles, and part of the bladder to account for minor filling uncertainties. For CK-SBRT with intrafraction imaging, a 3-5mm PTV was grown all around the GTV prostate, except for 2-3mm in posterior direction, taking care to be off the rectal wall. The pelvic nodal clinical target volume (CTV) was demarcated as per Radiation Therapy Oncology Group (RTOG) guidelines [14]. A 5mm three-dimensional margin was given around the gross nodes and the pelvic nodal CTV to generate the respective nodal PTVs. The bladder, rectum, bowel, femoral heads, pelvic bones and penile bulb were contoured as critical structures. The urethra was contoured on MRI, as described previously [15]. The dose prescription for VMAT in 25 fractions was 45 Gy to pelvic nodal PTV, 50 Gy to prostate PTV, and 54-56 Gy to gross nodal PTV; to be treated as five fractions per week. Bone oligometastases, if any, were treated with $16 \mathrm{~Gy}$ in two fractions, delivered on weekends. The dose prescription for CK-SBRT was $18 \mathrm{~Gy}$ in three fractions, prescribed to the $75-80 \%$ isodose covering the margin of prostate PTV. The dose-constraints for critical structures are mentioned in Table 1.

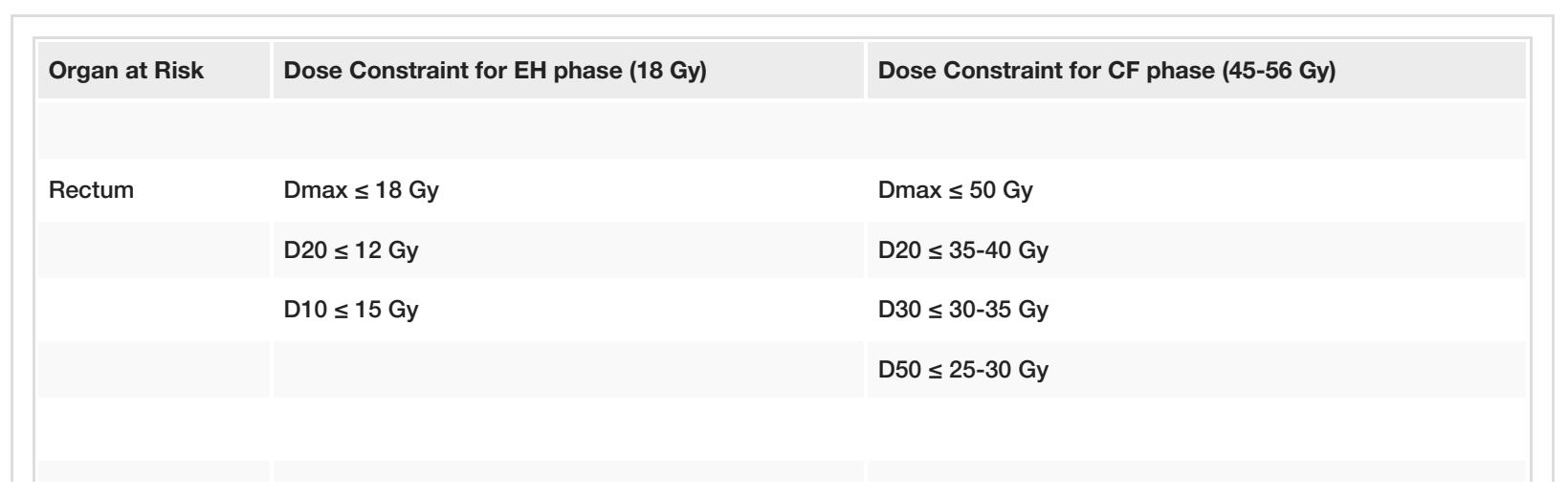




\section{Cureus}

\begin{tabular}{|c|c|c|}
\hline \multirow[t]{4}{*}{ Rectum - PTV } & $D \max \leq 18$ Gy & Dmax $\leq 45$ Gy \\
\hline & $\mathrm{D} 20 \leq 10 \mathrm{~Gy}$ & $\mathrm{D} 20 \leq 30-35 \mathrm{~Gy}$ \\
\hline & $\mathrm{D} 10 \leq 12 \mathrm{~Gy}$ & $\mathrm{D} 30 \leq 25-30$ Gy \\
\hline & & $D 50 \leq 20-25$ Gy \\
\hline \multirow[t]{4}{*}{ Bladder } & Dmax $\leq 18$ Gy & Dmax $\leq 50$ Gy \\
\hline & $\mathrm{D} 20 \leq 12$ Gy & D20 $\leq 40-45$ Gy \\
\hline & D10 $\leq 15$ Gy & D30 $\leq 35-40$ Gy \\
\hline & & D50 $\leq 25-30$ Gy \\
\hline \multirow[t]{4}{*}{ Bladder - PIV } & Dmax $\leq 18$ Gy & Dmax $\leq 45$ Gy \\
\hline & $\mathrm{D} 20 \leq 10 \mathrm{~Gy}$ & D20 $\leq 35-40$ Gy \\
\hline & D10 $\leq 14-16$ Gy & $D 30 \leq 30-35$ Gy \\
\hline & & D50 $\leq 20-25$ Gy \\
\hline \multirow[t]{2}{*}{ Bowel } & Dmax $\leq 10-15$ Gy & Dmax $\leq 45-50$ Gy \\
\hline & & D195cc $\leq 30-35$ Gy \\
\hline \multirow[t]{2}{*}{ Penile Bulb } & $\mathrm{D} 2 \mathrm{cc} \leq 15 \mathrm{~Gy}$ & Dmax $\leq 50$ Gy \\
\hline & & Dmean $\leq 30$ Gy \\
\hline Urethra & Dmax $\leq 18$ Gy & Dmax $\leq 50$ Gy \\
\hline Femur Heads & $\mathrm{D} 10 \leq 6 \mathrm{~Gy}$ & D50 $\leq 20$ Gy \\
\hline Pelvic Bones & D20 $\leq 8-10$ Gy & D50 $\leq 20$ Gy \\
\hline Genitalia & D10 $\leq 4$ Gy & Dmean $\leq 5$ Gy \\
\hline Thecal sac & & Dmax $\leq$ \\
\hline
\end{tabular}

\section{TABLE 1: Dose constraints for planning}

PTV: Planning Target Volume, EH: Extreme Hypofractionation, CF: Conventional Fractionation, Dmax: Maximum dose, Dmean: Mean Dose

D195cc: Dose to 195 cc of the structure, and so on

D20: Dose to $20 \%$ volume of the structure, and so on

Treatment Plan Generation and Evaluation

VMAT planning was carried out on Elekta Monaco ${ }^{\circledR}$ (Elekta, Stockholm, Sweden) treatment planning system, version 5.1.1. Two-stage optimization was performed using inverse planning. The constrained and Pareto optimization algorithm performed the gradient search for the most feasible solution to achieve acceptable target coverage with optimal organ sparing, followed by Monte Carlo algorithm for final dose calculation using $2 \mathrm{~mm}$ grid size. Two complete arcs (360 degrees), with arc length starting from 180 degrees to -180 degrees were most commonly used. For PTV, the goal was to deliver $>98 \%$ of the prescribed dose to $>95 \%$ of the volume, while maintaining maximum possible dose homogeneity, and respecting normal tissue constraints. Plan specific quality assurance was performed to verify parameters like gantry angle, multi-leaf collimator (MLC) shape and dose rate before treatment delivery for each patient. CK-SBRT planning was 
carried out on Accuray MultiPlan ${ }^{\circledR}$ treatment planning system, version 4.6.0. The initial step was tracking the implanted fiducials to the tracking synchrony of target localization. A minimum of three fiducials' definition was necessary for correlating the translational and rotational prostate variation during beam delivery. Inter-fiducial distance of $>2 \mathrm{~cm}$ and angulation $>15^{\circ}$ was ideal for achieving optimal correction strategy. Treatment anatomy of body and prostate path set were chosen to define the robot movement around the patient surface in the predetermined cloud space, with each robot position on this cloud being the designated "node". Suitable collimator sizes were chosen as per the target dimension. Limitation of monitor units (MU) for each beam and each node was set to achieve beam stability and correction of setup errors. Eight conformity shells were created surrounding the target to achieve target dose conformity and reduction of normal tissue doses. The gradient search sequential optimization algorithm then performed the dose optimization based on the dose constraints and objectives. After achieving an optimal plan, treatment time reduction was performed by optimizing robot movement, and beam reduction was done to reduce the lesser MU beams that were causing beam delivery instability. Subsequently, the final dose calculation was performed by using the ray tracing algorithm with a grid size of $1 \mathrm{~mm}$. The goal was to cover $>98 \%$ of the prostate PTV with the prescribed dose, while concentrating hot-spots within the prostate GTV. Care was taken to avoid hot-spots over the urethra and to reduce the irradiated volume of the penile bulb, rectal wall, and bladder neck, while respecting all other organ constraints. After plan approval, quality assurance was performed in phantom for that specific plan to verify all parameters like robot position, MU delivery, and dose accuracy before proceeding for treatment delivery. The bone oligometastatic site was treated with SBRT or less conformal techniques as deemed feasible.

\section{Treatment Delivery}

In the initial years, the CK-SBRT phase utilizing EH succeeded the VMAT based CF phase by approximately two weeks. An internal audit showed difficulties in delineating the outer surface of the prostate, and also greater fiducial tracking uncertainties with this approach. The former could probably be due to radiationinduced inflammation and the latter due to a greater time-gap between fiducial placement and treatment on CK. Hence, we changed our practice to treat the CK-SBRT phase first, followed by VMAT in the following week (reverse-boost approach). For CK-SBRT, patients were treated on empty bladder to maintain the geometry of implanted fiducials for proper tracking and to avoid discomfort to the patient during the long treatment fraction. Intrafraction imaging helped identify and correct translational and rotational displacements in real-time. The CK system utilizes a 6MV Flattening-Filter-Free (FFF) beam with a dose rate of $1000 \mathrm{MU} / \mathrm{min}$. Treatment fractions were delivered on an alternate-day basis, with each fraction lasting about 30-40 minutes. For VMAT, all patients were treated with dedicated bladder filling and rectal emptying protocol with daily bowel preparation, under stringent daily image guidance on Elekta Infinity ${ }^{\circledR}$ using X-ray volumetric imaging (XVI). Translational and rotational errors were corrected by means of Hexapod couch. A 6MV beam utilizing a dose rate of 200-400 MU/min was used. Each treatment fraction typically lasted for about seven to 10 minutes. Patients were reviewed daily during RT for ensuring appropriate execution of treatment delivery as per institutional standards and for recording and treating acute toxicity.

\section{Follow-up evaluation}

After RT completion, patients were followed-up initially after four to six weeks, and subsequently after every three to four months for the first two years, followed by every six months for the next three years, and annually thereafter. Serum PSA was done three-monthly for two years, and six-monthly thereafter. MRI pelvis or PSMA-PET were performed at six months post-RT, and annually thereafter. HT was continued for at least three years, or till a sustained PSA level of $0.02 \mathrm{ng} / \mathrm{ml}$ was attained across three successive six-monthly readings, whichever was later. Patients were prescribed bisphosphonate preparations to maintain bone health while on HT, and bone mineral density was checked annually. Acute and late toxicity was recorded as per Common Toxicity Criteria for Adverse Events, version 4.0 [16]. Progression was defined as local, regional or distant progression of disease on clinical examination or imaging, after due confirmation by cytology or biopsy from any of the progressed site(s). A biochemical progression was deemed to have occurred in case the PSA increased $2 \mathrm{ng} / \mathrm{ml}$ above the nadir PSA (nPSA $+2 \mathrm{ng} / \mathrm{ml}$ ) in accordance with the Phoenix modification of ASTRO definition [17]. The progressed disease was appropriately managed by either changing or restarting HT, switching to chemotherapy, or palliative radiation therapy.

\section{Statistical analysis}

Data tabulation and basic statistics were performed using Microsoft Excel ${ }^{\circledR} 2013$. The progression-free survival (PFS), biochemical progression free survival (bPFS), and overall survival (OS) were estimated through Kaplan-Meier survival statistics using MedCalc ${ }^{\circledR}$ software, version 19.5.2.

\section{Results}

\section{Patient characteristics}

Median age was 69 years (range 52-84 years) and median initial PSA (iPSA) was 26ng/ml (range 4.8$217 \mathrm{ng} / \mathrm{ml}$ ). Thirty-two (73\%) patients had GS 7 or 8 . There were 33/44 (75\%) locally advanced (T3a/bT4 N0), 9/44 (20.5\%) node-positive (N1), and 2/44 (4.5\%) bone oligometastatic (M1) cases. One oligometastatic 


\section{Cureus}

patient had a single D7 vertebral lesion and the other had two lesions in the left iliac bone. Patient characteristics are summarized in Table 2.

\begin{tabular}{|l|l|}
\hline Parameter & Value (Range) \\
\hline Age (years) & Median $=69(52-84)$ \\
\hline GS & Median $=8 \quad(6-9)$ \\
\hline 7 & 4 \\
\hline 8 & 17 \\
\hline 9 & 15 \\
\hline iPSA (ng/ml) & 8 \\
\hline Stage / Risk Group & Median $=26 \quad(4.8-217)$ \\
\hline T3a / High & Majority T3b / Very High \\
\hline T3b / Very High & 11 \\
\hline T4a / Very High & 20 \\
\hline N1 / Regional & 2 \\
\hline M1 / Metastatic & 9 \\
\hline Prostate Volume at baseline & 2 \\
\hline Prostate Volume before RT & Median = 45.9 cc (23- $104.8 \mathrm{cc})$ \\
\hline
\end{tabular}

\section{TABLE 2: Patient Characteristics}

GS: Gleason's Score, iPSA: initial PSA, RT: Radiation Therapy

Median follow-up, as calculated from initiation of neoadjuvant HT, was 63.5 months (range 17-92 months) and median HT duration was 31.5 months (range eight to 80 months), with the overall HT compliance in terms of taking HT till the prescribed duration being $86.4 \%$ (38/44 patients). The median duration of neoadjuvant HT was three months (range one to 10 months). The planning dose-constraints were achieved in all patients, and all patients could complete the full course of pelvic and prostate RT. The initial 15 (34\%) patients received $\mathrm{EH}$ boost after the $\mathrm{CF}$ course, and the remaining 29 (66\%) patients received it prior (reverse-boost approach).

\section{Outcomes}

The median nPSA was $0.01 \mathrm{ng} / \mathrm{ml}$ (range $0.002-0.8 \mathrm{ng} / \mathrm{ml}$ ), and the median time to reach nPSA was 13.5 months post-RT (range three to 63 months). Disease progression was noted in four patients, of which two had local and two had distant progression. Both patients experiencing local progression belonged to the Very-High risk category (T3b/T4a, NOM0). Of the patients experiencing distant progression, one patient belonged to the Very-High risk category (T3bN0M0) and one was oligometastatic (T3bN1M1) at baseline. The treated oligometastatic bone lesions were controlled on long-term follow-up. The five-year PFS was $88.2 \%$, bPFS was $91.4 \%$ and OS was $96.9 \%$ (Figure 1 ).
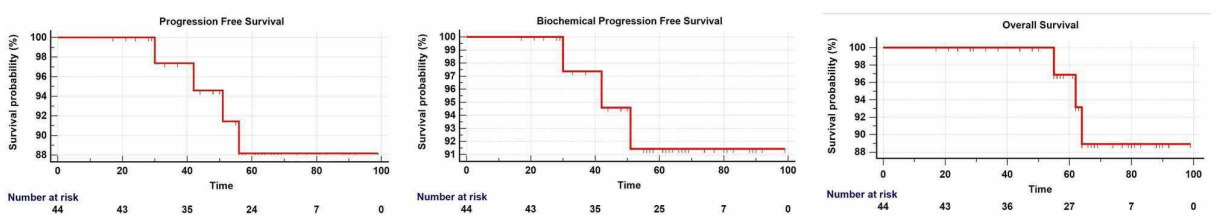

FIGURE 1: Progression-Free Survival, Biochemical Progression-Free Survival and Overall Survival 
Overall, mortality occurred in three patients, all from causes not related to PC. Mortality in two of the patients occurred after the five-year time point, and hence the six-year OS dropped to $89 \%$. Incidence of acute genito-urinary (GU) and gastrointestinal (GI) toxicity was $2.3 \%$ each. One patient developed grade 3 bladder outlet obstruction, requiring channel TURP within three months post-RT. One patient had per rectal bleed from internal haemorrhoids for which RT had to be temporarily withheld and the bleed was managed with Argon Laser photocoagulation. Two patients developed urethral strictures requiring dilatation at more than two years post-RT (Grade III late GU toxicity $=4.5 \%$ ). No patient developed Grade III late GI toxicity. HT related toxicity included gynaecomastia, andropausal behavioral symptoms, and osteoporotic vertebral collapse in one patient each.

\section{Discussion}

This series is unique in that it exclusively consists of loco-regionally advanced and oligometastatic PC, unlike previously published studies of $\mathrm{EH}$ boost or of $\mathrm{EH}$ alone that report outcomes of localized and advanced PC together. Secondly, the oligometastatic cases in our study were treated with a more radical intent and received the same treatment as non-metastatic cases. The prostate $(\alpha / \beta=1.5)$ received a BED of $206.7 \mathrm{~Gy}$, which translates into a 2-Gy Equivalent (EQD2) of $88.6 \mathrm{~Gy}$. The gross nodes $(\alpha / \beta=1.5)$ received BED between 131.8 to $139.6 \mathrm{~Gy}$ (EQD2 56.5 to $59.8 \mathrm{~Gy}$ ) and the nodal region $(\alpha / \beta=1.5)$ received BED $99 \mathrm{~Gy}$ (EQD2 $43.2 \mathrm{~Gy}$ ). Bladder and rectum $(\alpha / \beta=3$ ) received a maximum BED of $137.3 \mathrm{~Gy}$, with an EQD2 of 82.4 Gy, and bowel $(\alpha / \beta=3)$ received maximum BED of $72 \mathrm{~Gy}$ (EQD2 $43.2 \mathrm{~Gy})$.

Zaorsky et al., in their meta-analysis of conventional and hypofractionated schedules [18], demonstrated that BED of around $200 \mathrm{~Gy}$ to prostate is important for attaining favourable long-term control rates, and restricting critical structure BED around 133 Gy helps limit normal tissue toxicity. While this is easier for capsule-confined localized PC, dose-escalation for more advanced disease extending into neurovascular bundle or seminal vesicles, results in potentially toxic doses being delivered to bladder and rectum. Hence, a stricter balance between delivery of optimum BED and limiting normal tissue toxicity needs to be attained. We irradiated the pelvic nodal region in all cases, based on the RTOG 9413 trial that demonstrated the benefit of this approach for high-risk PC [19]. Doses $\geqslant 60$ Gy equivalent have been shown to be sufficient for gross nodal disease control [20]. Wang et al. [21] determined that a median SBRT dose of 37.4 Gy in four fractions to the rectum ( $\mathrm{BED}=154 \mathrm{~Gy} ; \alpha / \beta=3$ ) was associated with lesser late GI toxicity. Our treatment schedule complies with the doses and limits set by the above authors. The compliance to HT in terms of appropriate duration as per risk-category was $86.4 \%$. Two out of the four patients who progressed had interrupted HT due to treatment-related side-effects. With this approach, we obtained a five-year bPFS of $91.4 \%$, OS of $96.9 \%$ and $\geqslant$ Grade 3 late toxicity of $4.5 \%$ at a median follow-up of 63.5 months, which are better than other EH series utilizing a similar treatment approach (Table 3). 


\begin{tabular}{|c|c|c|c|c|c|c|}
\hline Author & $\mathbf{n}$ & Stage / Risk & Fractionation, HT use & Median FU & Survival & $\begin{array}{l}\geq \text { Gr } 3 \\
\text { Toxicity }\end{array}$ \\
\hline Miralbell [5] & 50 & $\begin{array}{l}\text { Localised - 30, } \\
\text { Advanced - } 20 \\
\quad \text { Low - 5, Int - } \\
\text { 12, High - } 33\end{array}$ & $\begin{array}{l}\text { Prostate RT 64-64.4 Gy in } 32-35 \mathrm{fr} \\
\text { (3DCRT / IMRT / VMAT), followed } \\
\text { by EH boost 10-16 Gy in } 2 \mathrm{fr} \text { (IMRT) } \\
\text { to dominant tumour nodule HT - } \\
33 \text { pts }\end{array}$ & 63 months & 5-yr bPFS 98\% & $\begin{array}{l}\text { GU : Ac } \\
4 \% \text {, Late } \\
0 \% \quad \text { GI } \\
\text { : Ac } 0 \% \\
\text { Late } 10 \%\end{array}$ \\
\hline Lin [6] & 41 & $\begin{array}{l}\text { Localised - } 24 \text {, } \\
\text { Advanced - } 17 \text { High - } \\
32, \text { V. High - } 9\end{array}$ & $\begin{array}{l}\text { Pelvic RT } 45 \text { Gy in } 25 \mathrm{fr}+ \\
\text { EH boost to prostate } 21 \text { Gy in } 3 \mathrm{fr} \\
\text { HT - } 38 \text { pts }\end{array}$ & 42 months & $\begin{array}{l}4-y r \text { bPFS } \\
91.9 \% \quad 4-y r \text { OS } \\
92.2 \%\end{array}$ & $\begin{array}{l}\text { GU : Ac } \\
0 \% \text {, Late } \\
0 \% \quad \text { GI } \\
: \text { Ac } 0 \% \\
\text { Late } 0 \%\end{array}$ \\
\hline Freeman [7] & 1743 & $\begin{array}{l}\text { Localised \& Advanced } \\
\text { Low - 819, Int - } 619 \text {, } \\
\text { High - 168, V. High - } 4 \\
\text { Metastatic - } 3 \\
\text { Unspecified - } 130\end{array}$ & $\begin{array}{l}35-40 \text { Gy in } 5 f r \text { (Monotherapy) - } \\
1499 \text { pts Pelvic RT } 45-50 \text { Gy + } \\
\text { Boost 19.5-21.75 Gy in } 3 \mathrm{fr}-139 \\
\text { pts }\end{array}$ & 24 months & $\begin{array}{c}2 \text { yr bPFS } 92 \% \\
\quad \text { Low - } \\
99 \%, \quad \text { Int } \\
\text { (GS 3+4) }-97 \% \\
\text { Int (GS } 4+3)- \\
85 \% \quad \text { High - } \\
87 \%\end{array}$ & $\begin{array}{l}\text { GU : Late : } \\
0 \% \quad \text { GI } \\
: \text { Late : } \\
0.1 \%\end{array}$ \\
\hline Anwar [8] & 48 & $\begin{array}{l}\text { Localized - } 28 \text {, } \\
\text { Advanced - } 20 \text { Int - } \\
14, \text { High - } 34\end{array}$ & $\begin{array}{l}\text { Pelvic RT } 45 \text { Gy in } 25 \mathrm{fr}+\mathrm{SBRT} \\
\text { boost } 17-21 \mathrm{~Gy} \text { in } 2 \mathrm{fr} \text { HT }-45 \text { pts }\end{array}$ & 42.7 months & $\begin{array}{l}\text { 5-yr PFS } 83 \% \\
5-y r \text { bPFS } \\
90 \%\end{array}$ & $\begin{array}{l}\text { GU: Ac: } 0 \% \text {, } \\
\text { Late: } 2.1 \% \\
\text { GI : Ac : } \\
0 \% \text {, Late : } \\
0 \%\end{array}$ \\
\hline $\begin{array}{l}\text { Mercado [9] } \\
\text { Paydar [10] }\end{array}$ & 108 & $\begin{array}{l}\text { Localised - 107, } \\
\text { Advanced - } 1 \text { Low - } 4 \text {, } \\
\text { Int - } 45 . \text { High - } 59\end{array}$ & $\begin{array}{l}\text { SBRT } 19.5 \text { Gy in } 3 \mathrm{fr}, \mathrm{f} / \mathrm{b} \text { Pelvic } \\
\text { IMRT } 45-50.4 \text { Gy in } 25-28 \text { fr HT - } \\
70 \text { pts }\end{array}$ & 53 months & $\begin{array}{l}\text { 3-yr bPFS } \\
100 \% \quad 3-y r \\
\text { bPFS } 89.8 \%\end{array}$ & $\begin{array}{l}\mathrm{GU}: \mathrm{Ac}: \\
1 \%, \text { Late }: \\
6.5 \% \mathrm{Gl}: \\
\text { Ac : } 0 \% \\
\text { Late }: 1 \%\end{array}$ \\
\hline $\operatorname{Kim}$ [11] & 39 & $\begin{array}{l}\text { Localised - 35, } \\
\text { Advanced - } 4 \text { Int - 20, } \\
\text { High - } 19\end{array}$ & $\begin{array}{l}\text { Pelvic RT } 45 \text { Gy in } 25 \mathrm{fr}+\mathrm{SBRT} \\
\text { boost } 21 \text { Gy in } 3 \text { fr No HT }\end{array}$ & 53.6 months & $\begin{array}{l}5-y r b P F S \\
94.7 \%\end{array}$ & $\begin{array}{l}\text { GU : Ac : } \\
0 \%, \text { Late : } \\
0 \% \text { GI : Ac } \\
: 0 \%, \text { Late : } \\
0 \%\end{array}$ \\
\hline $\begin{array}{l}\text { Pryor } \\
\text { (PROMETHEUS } \\
\text { Trial) [12] }\end{array}$ & 135 & $\begin{array}{l}\text { Localised \& Advanced } \\
\text { Int - 103, High - } 32\end{array}$ & $\begin{array}{l}\text { GBRT } 19-20 \text { Gy in } 2 \mathrm{fr}, \mathrm{f} / \mathrm{b} \text { IMRT } 46 \\
\text { Gy in } 23 \mathrm{fr} \text { or } 36 \text { Gy in } 12 \mathrm{fr} \\
-73 \text { pts }\end{array}$ & 24 months & $\begin{array}{l}2-y r \text { bPFS } \\
98.6 \%\end{array}$ & $\begin{array}{l}\text { GU : Ac : } \\
0 \%, \text { Late : } \\
2.2 \% \text { Gl : } \\
\text { Ac : } 0 \% \\
\text { Late }: 2 \%\end{array}$ \\
\hline This report & 44 & $\begin{array}{l}\text { All Advanced : High - } \\
11, \text { V. High - 22, N1 - } \\
9, \text { M1 - } 2\end{array}$ & $\begin{array}{l}\text { VMAT } 50 \text { Gy, f/b CK SBRT } 18 \text { Gy in } \\
3 \text { fr HT - All pts }\end{array}$ & 63.5 months & $\begin{array}{l}5-y r \text { PFS } 88.2 \% \\
5 \text {-yr bPFS } \\
91.4 \% 5-y r \text { OS } \\
96.9 \%\end{array}$ & $\begin{array}{l}\text { GU : Ac } \\
2.3 \%, \text { Late } \\
4.5 \% \text { Gl : } \\
\text { Ac } 2.3 \% \\
\text { Late } 0 \%\end{array}$ \\
\hline
\end{tabular}

\section{TABLE 3: Outcomes with EH boost for High-Risk Advanced PC}

SBRT: Stereotactic Body Radiation Therapy, IMRT: Intensity Modulated Radiation Therapy, 3DCRT: 3-dimensional Conformal Radiation Therapy, VMAT: Volumetric Modulated Arc Therapy, EH: Extreme Hypofractionation, Ac: Acute

Lin et al. [6] treated 41 high-very high-risk cases, of which 17 (41\%) were advanced PC, with pelvic RT and EH boost of $21 \mathrm{~Gy}$ in three fractions to the prostate. At a median follow-up of 42 months, they reported a four-year bPFS of $91.9 \%$ and OS of $92.2 \%$. In the registry of 743 patients by Freeman et al. [7], 175 (24\%) belonged to high-very high and oligometastatic category. The combination of pelvic RT, along with prostate boost 19.5 to 21.75 Gy in three fractions was utilized in 139 (19\%) patients. A two-year bPFS of $87 \%$ was reported. Anwar et al. [8] treated 48 patients, of which 34 (71\%) were high-risk and 20 (42\%) were advanced PC, with pelvic RT and SBRT boost of 17-21 Gy in two fractions to the prostate. At a median follow-up of 42.7 months, they reported a five-year bPFS of 90\% and five-year PFS of 83\%. In the PROMETHEUS trial [12], 135 
localized and advanced PC cases, including 32 (24\%) high-risk patients, were treated with SBRT 19-20 Gy in two fractions to prostate, followed by pelvic IMRT $46 \mathrm{~Gy}$ in 23 fractions or $36 \mathrm{~Gy}$ in 12 fractions. HT was used in 73 patients. A two-year bPFS of $98.6 \%$ was reported.

Among studies employing EH alone for advanced PC, Murthy et al. [22] treated 68 high-very high risk and node-positive cases, of which 50 (74\%) were advanced PC, using tomotherapy-based IMRT to doses of 35$37.5 \mathrm{~Gy}$ to prostate and $25 \mathrm{~Gy}$ to nodes in five fractions, along with HT for at least two years in all patients. They reported a combined 1.5 -year bPFS of $94 \%$ and OS of $97 \%$. Although this study meets the radiobiological doses and limits criteria described above, the authors need to report much longer follow-up data to be of relevance. The HYPO-RT PC trial [23] included 1180 intermediate- and high-risk cases, of which 51 (4\%) were advanced PC, and similar five-year bPFS of $84 \%$ were obtained in both arms of EH 42.7 Gy in seven fractions and CF 78 Gy in 39 fractions. The proportion of advanced PC cases was severely limited in this trial. Despite this, our five-year outcomes are clearly superior to those reported in this trial.

Most series of advanced PC patients with reported median follow-up duration $>$ four years have late $\geqslant$ Grade $3 \mathrm{GU}$ and GI toxicity rates up to $6.5 \%$ and $3.3 \%$, respectively [10-11]. Our toxicity rates are comparable to these. Efficient RT planning, use of dedicated rectal emptying and bladder filling protocols, omission of uninvolved portion of the seminal vesicles from the target volume, limiting of posterior PTV margins to limit the rectal volume being irradiated, delineation of urethra and limiting hot-spots inside it, daily imageguidance for VMAT, intrafraction motion tracking on CK by use of implanted fiducials, alternate-day treatment schedule for CK-SBRT, and delivering CK-SBRT before VMAT are some of the factors responsible for limiting late toxicity. A study by Feng et al. [24] compared late GU toxicity between two schedules of SBRT alone versus a combination of IMRT and SBRT boost similar to our study and found lesser toxicity with the latter approach. Patients with prostate volume $>50 \mathrm{cc}$ at baseline have been shown to experience greater GU toxicity [25]. In our study, the median prostate volume was 43.9 cc before neoadjuvant HT and 23.5 cc before RT. Two of our patients (4.5\%) developed urethral strictures. This agrees with the results by Awad et al. [26], who demonstrated pooled estimate prevalence of urethral strictures of $1.5 \%$ for external beam RT alone, $1.9 \%$ for brachytherapy alone, and $4.9 \%$ for a combination of both.

Efforts to further improve outcomes using different approaches for advanced and oligometastatic PC are ongoing. SBRT dose escalation to the dominant intraprostatic nodule as seen on MRI or PSMA-PET has been reported [5], with good short-term control rates. The NINJA (TROG 18.01) [27] is an extension of the PROMETHEUS trial [12] and compares SBRT boost $20 \mathrm{~Gy}$ in two fractions followed by pelvic RT 36 Gy in 12 fractions to SBRT $36.25 \mathrm{~Gy}$ (PTV) and $40 \mathrm{~Gy}$ (CTV) in five fractions. The PBS trial [28] is investigating toxicity and quality-of-life in advanced PC patients treated with pelvic RT 45-46 Gy followed by boost 32-33 Gy with CF or 19-21 Gy in three fractions with SBRT. The PRIME trial [29] compares EH 36.25 Gy in five fractions over seven to 10 days against the standard MH 68 Gy in 25 fractions over five weeks for high-very high and node-positive PC. Results of these trials shall help determine the most suitable hypofractionated schedule for advanced PC.

The idea of irradiating the primary tumour and the oligometastatic disease sites has been a subject of randomized trials in recent years. The STAMPEDE trial [30] randomized metastatic PC patients to standard of care HT or chemotherapy arms versus the experimental arm additionally incorporating RT to the prostate. The RT was either an MH schedule of $55 \mathrm{~Gy}$ in 20 fractions or an EH schedule of $36 \mathrm{~Gy}$ in six fractions, oncea-week delivery. Although there was no improvement in OS, the RT arm showed a statistically significant improvement in failure-free survival (HR 0.76, 95\% CI 0.68-0.84; $<$ < 0.0001). Results of the ongoing ORIOLE, FORCE, and PEACE1 trials shall further clarify the role of RT in oligometastatic PC.

Despite the good five-year outcomes, we do admit that our study has limitations of non-randomized design, relatively smaller patient numbers and follow-up duration. Since long-term tumour control is a combined effect of HT and RT, quantifying the individual contribution of RT dose-escalation by means of EH may be difficult. We do not believe that sequencing of the EH boost may alter disease outcomes, since the total BED is similar. The overall treatment time (OTT) does differ, being eight weeks in patients who received EH boost after the CF course, and six weeks in those who received it prior. This way, the reverse boost approach offers twin benefits of EH as well as reduction in OTT as compared to conventional and sequential boost regimens. As such, our patient numbers are too small to detect any difference in outcomes based on the differences in OTT, if any. It is worthwhile to try an appropriately adjusted combination of $\mathrm{MH}$ and $\mathrm{EH}$ for abbreviating the OTT further for this specific cohort of advanced and metastatic PC.

\section{Conclusions}

Our phase I/II study proves that utilizing EH as a component of treatment for locally advanced, nodepositive and oligometastatic PC, along with appropriate duration HT, can help attain excellent five-year control and survival rates, along with acceptable toxicity. This merits testing EH against a CF or MH treatment arm in a Phase III randomized trial. The anatomical location of the prostate and the biology of prostate cancer places it in a favorable situation where EH schedules are likely to enhance the therapeutic ratio further. With an increasing number of ongoing trials focusing on radical local treatment strategies for oligometastatic disease, the future appears bright for extending the domain of RT in this disease setting. Results of these trials shall help determine the best hypofractionated schedule for advanced and 


\section{Additional Information Disclosures}

Human subjects: Consent was obtained by all participants in this study. Medanta Institutional Review Board, Medanta The Medicity, Sector 38, Gurugram, IND issued approval NA. The present study was conducted in accordance with the guidelines laid down by the Medanta Institutional Review Board and Institutional Ethics Committee. No funding was required for the study. Animal subjects: All authors have confirmed that this study did not involve animal subjects or tissue. Conflicts of interest: In compliance with the ICMJE uniform disclosure form, all authors declare the following: Payment/services info: All authors have declared that no financial support was received from any organization for the submitted work. Financial relationships: All authors have declared that they have no financial relationships at present or within the previous three years with any organizations that might have an interest in the submitted work. Other relationships: All authors have declared that there are no other relationships or activities that could appear to have influenced the submitted work.

\section{References}

1. Dearnaley DP, Jovic G, Syndikus I, et al.: Escalated-dose versus control-dose conformal radiotherapy for prostate cancer: long-term results from the MRC RT01 randomised controlled trial. Lancet Oncol. 2014, 15:464-473. 10.1016/S1470-2045(14)70040-3

2. Dearnaley DP, Syndikus I, Mossop H, et al.: Conventional versus hypofractionated high-dose intensitymodulated radiotherapy for prostate cancer: 5-year outcomes of the randomised, non-inferiority, phase 3 CHHiP trial. Lancet Oncol. 2016, 17:1047-1060. 10.1016/S1470-2045(16)30102-4

3. de Vries KC, Wortel RC, Oomen-de Hoop E, Heemsbergen WD, Pos FJ, Incrocci L: Hyprofractionated versus conventionally fractionated radiation therapy for patients with intermediate- or high-risk, localized, prostate cancer: 7-year outcomes from the randomized, multicenter, open-label, phase 3 HYPRO trial. Int J Radiat Oncol Biol Phys. 2020, 106:108-115. 10.1016/j.ijrobp.2019.09.007

4. Lukka HR, Pugh SL, Bruner DW, et al.: Patient reported outcomes in NRG Oncology RTOG 0938, evaluating two ultrahypofractionated regimens for prostate cancer. Int J Radiat Oncol Biol Phys. 2018, 102:287-295. 10.1016/j.ijrobp.2018.06.008

5. Miralbell R, Mollà M, Rouzaud M, et al.: Hypofractionated boost to the dominant tumor region with intensity modulated stereotactic radiotherapy for prostate cancer: a sequential dose escalation pilot study. Int J Radiat Oncol Biol Phys. 2010, 1:50-57. 10.1016/j.ijrobp.2009.07.1689

6. Lin YW, Lin LC, Lin KL: The early result of whole pelvic radiotherapy and stereotactic body radiotherapy boost for high-risk localized prostate cancer. Front Oncol. 2014, 31:278. 10.3389/fonc.2014.00278

7. Freeman D, Dickerson G, Perman M: Multi-institutional registry for prostate cancer radiosurgery: a prospective observational clinical trial. Front Oncol. 2015, 22:369. 10.3389/fonc.2014.00369

8. Anwar M, Weinberg V, Seymour Z, Hsu IJ, Roach M 3rd, Gottschalk AR: Outcomes of hypofractionated stereotactic body radiotherapy boost for intermediate and high-risk prostate cancer. Radiat Oncol. 2016, 21:8. 10.1186/s13014-016-0585-y

9. Mercado C, Kress MA, Cyr RA, et al.: Intensity-modulated radiation therapy with stereotactic body radiation therapy boost for unfavorable prostate cancer: the Georgetown University experience. Front Oncol. 2016, 6:114. 10.3389/fonc.2016.00114

10. Paydar I, Pepin A, Cyr RA, et al.: Intensity-modulated radiation therapy with stereotactic body radiation therapy boost for unfavorable prostate cancer: a report on 3-year toxicity. Front Oncol. 2017, 7:5. 10.3389/fonc.2017.00005

11. Kim HJ, Phak JH, Kim WC: Clinical outcomes of whole pelvis radiotherapy and stereotactic body radiotherapy boost for intermediate- and high-risk prostate cancer. Asia Pac J Clin Oncol. 2017, 13:342-347. 10.1111/ajco.12455

12. Pryor D, Sidhom M, Arumugam S, et al.: Phase 2 multicenter study of gantry-based stereotactic radiotherapy boost for intermediate and high risk prostate cancer (PROMETHEUS). Front Oncol. 2019, 2:217. 10.3389/fonc.2019.00217

13. NCCN Clinical Practice Guidelines in Oncology. https://www.nccn.org/professionals/physician_gls/default.aspx\#prostate.

14. Lawton CA, Michalski J, El-Naqa I, et al.: RTOG GU radiation oncology specialists reach consensus on pelvic lymph node volumes for high-risk prostate cancer. Int J Radiat Oncol Biol Phys. 2009, 1:383-387. 10.1016/j.ijrobp.2008.08.002

15. Kataria T, Gupta D, Goyal S, et al.: Simple diagrammatic method to delineate male urethra in prostate cancer radiotherapy: an MRI based approach. Br J Radiol. 2016, 89:20160348. 10.1259/bjr.20160348

16. Common terminology criteria for adverse events (CTCAE) version 4.0 . (2009). https://evs.nci.nih.gov/ftp1/CTCAE/CTCAE_4.03/Archive/CTCAE_4.0_2009-0529_QuickReference_8.5x11.pdf.

17. Roach M III, Hanks G, Thames H Jr, et al.: Defining biochemical failure following radiotherapy with or without hormonal therapy in men with clinically localized prostate cancer: recommendations of the RTOGASTRO Phoenix consensus conference. Int J Radiat Oncol Biol Phys. 2006, 65:965-974. 10.1016/j.ijrobp.2006.04.029

18. Zaorsky NG, Palmer JD, Hurwitz MD, Keith SW, Dicker AP, Den RB: What is the ideal radiotherapy dose to treat prostate cancer? A meta-analysis of biologically equivalent dose escalation. Radiother Oncol. 2015, 115:295-300. 10.1016/j.radonc.2015.05.011

19. Roach M 3rd, DeSilvio M, Lawton C, et al.: Phase III trial comparing whole-pelvic versus prostate-only 
radiotherapy and neoadjuvant versus adjuvant combined androgen suppression: Radiation Therapy Oncology Group 9413. J Clin Oncol. 2003, 21:1904-1911. 10.1200/JCO.2003.05.004

20. Engels B, Soete G, Tournel K, et al.: Helical tomotherapy with simultaneous integrated boost for high-risk and lymph-node positive prostate cancer: early report on acute and late toxicity. Technol Cancer Res Treat. 2009, 8:353-359. 10.1177/153303460900800505

21. Wang K, Chen RC, Kane BL, et al.: Patient and dosimetric predictors of genitourinary and bowel quality of life after prostate SBRT: secondary analysis of a multi-institutional trial. Int J Radiat Oncol Biol Phys. 2018, 102:1430-1437. 10.1016/j.ijrobp.2018.07.191

22. Murthy V, Gupta M, Mulye G, et al.: Early results of extreme hypofractionation using stereotactic body radiation therapy for high-risk, very high-risk and node-positive prostate cancer. Clin Oncol (R Coll Radiol). 2018, 30:442-447. 10.1016/j.clon.2018.03.004

23. Widmark A, Gunnlaugsson A, Beckman L, et al.: Ultra-hypofractionated versus conventionally fractionated radiotherapy for prostate cancer: 5-year outcomes of the HYPO-RT-PC randomised, non-inferiority, phase 3 trial. Lancet. 2019, 394:385-395. 10.1016/S0140-6736(19)31131-6

24. Feng LR, Suy S, Collins SP, Lischalk JW, Yuan B, Saligan LN: Comparison of late urinary symptoms following SBRT and SBRT with IMRT supplementation for prostate cancer. Curr Urol. 2018, 11:218-224. 10.1159/000447222

25. Aizer AA, Anderson NS, Oh SC, Yu JB, McKeon AM, Decker RH, Peschel RE: The impact of pretreatment prostate volume on severe acute genitourinary toxicity in prostate cancer patients treated with intensitymodulated radiation therapy. Int J Radiat Oncol Biol Phys. 2011, 79:379-84. 10.1016/j.ijrobp.2009.11.023

26. Awad MA, Gaither TW, Osterberg EC, Murphy GP, Baradaran N, Breyer BN: Prostate cancer radiation and urethral strictures: a systematic review and meta-analysis. Prostate Cancer Prostatic Dis. 2018, 21:168-174. 10.1038/s41391-017-0028-3

27. Martin J, Keall P, Siva S, et al.: TROG 18.01 phase III randomised clinical trial of the novel integration of new prostate radiation schedules with adjuvant androgen deprivation: NINJA study protocol. BMJ Open. 2019, 9:030731. 10.1136/bmjopen-2019-030731

28. Isfahanian N, Lukka H, Dayes I, et al.: A randomized phase II trial of prostate boost irradiation with stereotactic body radiotherapy (SBRT) or conventional fractionation (CF) external beam radiotherapy (EBRT) in locally advanced prostate cancer: the PBS trial (NCT03380806). Clin Genitourin Cancer. 2020, 18:410-415. 10.1016/j.clgc.2019.12.020

29. Murthy V, Mallik I, Gavarraju A, et al.: Study protocol of a randomised controlled trial of prostate radiotherapy in high-risk and node-positive disease comparing moderate and extreme hypofractionation (PRIME TRIAL). BMJ Open. 2020, 10:034623. 10.1136/bmjopen-2019-034623

30. Parker CC, James ND, Brawley CD, et al.: Radiotherapy to the primary tumour for newly diagnosed, metastatic prostate cancer (STAMPEDE): a randomised controlled phase 3 trial. Lancet. 2018, 392:23532366. 10.1016/S0140-6736(18)32486-3 